\title{
The Form of Analog Size Information in Memory
}

\author{
KEITH J. HOLYOAK \\ University of Michigan
}

\begin{abstract}
The information used to choose the larger of two objects from memory was investigated in two experiments that compared the effects of a number of variables on the performance of subjects who either were instructed to use imagery in the comparison task or were not so instructed. Subjects instructed to use imagery could perform the task more quickly if they prepared themselves with an image of one of the objects at its normal size, rather than with an image that was abnormally big or small, or no image at all. Such subjects were also subject to substantial selective interference when asked to simultaneously maintain irrelevant images of digits. In contrast, when subjects were not specifically instructed to use imagery to reach their decisions, an initial image at normal size did not produce significantly faster decisions than no image, or a large or small image congruent with the correct decision. The selective interference created by simultaneously imaging digits was reduced for subjects not told to base their size comparisons on imagery. The difficulty of the size discrimination did not interact significantly with any other variable. The results suggest that subjects, unless specifically instructed to use imagery, can compare the size of objects in memory using information more abstract than visual imagery.
\end{abstract}

The problem of how information about continuous dimensions is coded in memory has been the focus of several recent experimental studies. Much of this interest has resulted from the possibility that memory representations of such dimensions may be continuous, in much the same way as we conceive of the dimensions themselves. For example, objects in the world can take on essentially continuous objective size values. It is true, of course, that human perception, and presumably memory as well, have limited resolution, so that two objects may vary in size by so little that a person cannot discriminate between them on that basis. It is also possible that at a physiological level, magnitude information is represented by processes involving discrete events, such as neural firing. Nevertheless, at the level of description relevant to a cognitive model, size information in memory might be best conceptualized as approximating a continuously varying analog measure.

Evidence that size information is analog in the above sense has been

This study is a portion of a Ph.D dissertation submitted to Stanford University. Gordon Bowcr, Herbert Clark, Stephen Kosslyn, Keith Patterson, Roger Shepard, and Edward Smith provided valuable comments. This research was supported by NIMH Grant MH13950-08 to Gordon Bower and NIMH Grant 20020 to Herbert Clark. Reprint requests should be sent to the author at University of Michigan, Human Performance Center, 330 Packard Road, Ann Arbor, MI 48104. 
provided by studies that have measured people's reaction time (RT) to decide from memory which of two objects is larger. The basic finding that has emerged is that RT in this task decreases as the objective size difference between the two objects is increased. This result was first obtained by Moyer (1973) using animal names. He found, for example, that people could decide that a horse is bigger than a roach more quickly than they could determine that a horse is bigger than a sheep. This result, replicated by Jamieson and Petrusic (1975), has been extended by Paivio (1975) to objects other than animals. Using artificial materials, Moyer and Bayer (1976) have provided evidence that decision time depends not simply on the ordinal difference between two objects, but on the difference between their absolute magnitudes.

This "symbolic distance effect," as it has been termed by Moyer and Bayer, has been found in a number of similar comparison tasks involving other dimensions. These include comparisons of digits (Banks, Fujii, \& Kayra-Stuart, in press; Buckley \& Gillman, 1974; Moyer \& Landauer, 1967; Parkman, 1971; Sekuler, Rubin, \& Armstrong, 1971) and of terms drawn from the natural-language scales of time, temperature, and quality (Holyoak \& Walker, 1976). A related distance effect has been obtained with arbitrary names taught to subjects as a linear order along some dimension (Potts, 1972, 1974). In all of these domains there is therefore evidence consistent with the view that the ease of discriminating between stored dimensional values reflects the subjective difference between the magnitudes of such values.

What remains unclear, however, is the exact nature of the information underlying the symbolic distance effects obtained in different domains. Do all involve analog representations of magnitude information? How similar is the form of information about different dimensions, such as size and numerical magnitude? While theorists have speculated that a common analog representational system may underlie different domains (Moyer \& Bayer, 1976), there is as yet no direct evidence for this view. The lack of a clear account of the form in which analog size information might be stored has been pointed out by Moyer: "A serious deficiency in this formulation is, of course, that the nature of the postulated analogue representations is not specified. They may be positions along an imagined spatial dimension, temporal patterns in neural ensembles, rich images, or an as yet unimagined possibility" (1973, p. 183).

Given this limited state of knowledge, it seems that a fairly detailed analysis of the possible forms of representation used in particular task domains may be a prerequisite to any general model of mental comparisons. The present study investigates the possible role of visual imagery in mental size comparisons. The size dimension was chosen because it offers a rich data source, since people know the size relations 
between a great many objects, and because the mechanisms underlying size comparisons may plausibly be expected to generalize at least to other physical dimensions such as length, height, and brightness. In addition, size comparisons are known to produce a clear distance effect. Visual imagery, while not the only possible mode of analog size representation (as argued below), has been suggested as a possible candidate by theorists such as Moyer. People commonly report images while making mental size comparisons, and while it is difficult to imagine how visual imagery could underlie all analog dimensional information (particularly for nonperceptual dimensions, such as intelligence or time), it remains a plausible possibility for the other physical dimensions most related to size. These considerations suggested that an investigation of imagery and alternatives to imagery in the domain of size comparisons might offer some insight into the general question of how continuous dimensional information is remembered.

Three possible views of the information used for size comparisons are outlined below, along with arguments in support of each. Two experiments that attempted to provide evidence discriminating among these views are then reported.

\section{Hypothesis 1: Imagery Is Always Used}

The hypothesis that imagery has a central role in the size comparison process was first suggested by the striking similarity of the functions relating RT and objective distance in mental size comparisons (Moyer, 1973) and in perceptual comparisons, such as comparisons of line lengths (Johnson, 1939). In both cases, the function seems to be approximately inversely linear with the logarithm of the difference in magnitude of the two objects being compared (Moyer, 1973). Moyer argues that this similarity suggests that mental size comparisons involve an "internal psychophysical judgment." Visual imagery would seem to provide a possible basis for such a judgment. When presented with the names of two objects, a person may retrieve images of each object from long-term memory, and then compare the size of the two images to make a decision. Since images are presumably analogs of the actual objects, this internal comparison process might be expected to show effects very similar to those found in actual perceptual comparisons.

This hypothesis invokes the notion of a processor that can scan images. Pylyshyn (1973) has argued that such a processor must be a mythical "mind's eye," and that it leads to an infinite regress. However, the notion of a device that can compare images seems no more mysterious than any other cxecutive processor postulated in information processing models. Kosslyn (1975) offers some suggestions as to how an image processor might operate.

Paivio (1975) has obtained an additional result which might appear 
to favor the imagery hypothesis. He found that size comparisons could be made more quickly when the stimuli were pictures rather than names of the items. Hypothesis 1 predicts this result, assuming that images are aroused more quickly by pictures than by words. The imagery hypothesis also clearly accounts for the intuition of many people that they experience visual imagery in the size comparison task.

\section{Hypothesis 2: Imagery Is Sometimes Used}

This second view could be formulated in various ways, but one possibility is that imagery plays a central role only in those size comparisons that require especially close discriminations. For example, consider a modified version of the model proposed by Banks et al. (1976) for comparisons of numerical magnitude. It could be that objects very diffcrent in size (e.g., elephant and mouse) are coded in different size categories (e.g., "large" and "small"). This categorical information would be sufficient to decide which object is larger. However, objects relatively close in size (e.g., wolf and $d o g$ ) would likely fall into the same size category. For pairs close in size categorical information will therefore not distinguish the relative size of the objects, so that it will be necessary for the person to retrieve more precise analog size information. If this analog information is in the form of visual images, it follows that visual imagery will be central to the comparison process only for object pairs relatively similar in size.

This hypothesis is consistent with the intuition of some people that they mainly experience visual imagery in the size comparison task when the discrimination is especially difficult. It suggests that the distance effect in the size comparison task may be partly due to qualitative processing differences - comparisons of objects close in size may require a different type of information than comparisons of objects very different in size.

\section{Hypothesis 3: Imagery Is Never Used}

This third position derives from two directions of argument. First, a closer examination of empirical evidence seems to indicate that known properties of images are inconsistent with their playing a central role in mental size comparisons; second, there are reasonably clear alternative views of how analog information might be remembered.

If people compare images to decide which of two objects are larger, one would expect that images must readily convey information about the typical size of objects. This would be the case if people directly retrieve from long-term memory images of objects at their canonical size. However, Kosslyn (1975) has shown that people are quickest not to form images of animals at their canonical size, but rather to form the smallest size of image possible. This result is not affected by the objective size 
of the animals (at least in the range of mouse to collie): Regardless of objective size, small images can be formed more quickly than larger ones. In addition, the simple fact that people can image objects at any size at all other than the canonical one seriously complicates the imagery hypothesis, since somehow it must ensure that both objects are imaged at the same scale before they are compared.

Kosslyn presents a variety of evidence supporting the view that images are not remembered as units, but rather are constructed in active memory on the basis of more abstract perceptual information in long-term memory. Palmer (1975) has proposed that our perceptual knowledge of common objects may be stored in the form of abstract structural descriptions of prototypical exemplars. The representation of an object in such a model consists of hierarchically organized subunits. While these structural units are discrete, they may have analog parameters attached to them to indicate values on continuous dimensions. For instance, analog parameters might indicate the length of a giraffe's neck, both relative to other subparts of the animal and on some absolute scale. Such abstract analog information could then be used to construct images of objects in active memory and to adjust two images to a common size scale. But in this view, the natural locus of the size comparison process is not images, but rather the underlying analog parameters of structural descriptions.

Note that this hypothesis is consistent with people's intuitions that they form images when making size comparisons. The abstract analog parameters may enter into image construction at the same time as they are used to compare sizes. It is even possible that instructing oneself to form images is an cfficient way to activate size information, even though a size comparison can then be made before the images are actually constructed. The time course of these two processes (image construction and magnitude comparison) may account for the intuition that images mainly accompany comparisons of pairs close in size. When the two objects are very different in size, the comparison will be relatively fast and usually will be completed before the objects are imaged. When the objects are similar in size, on the other hand, the comparison will be slower and may not be completed until after the objects have been imaged. The important point is that while people may form images when making size comparisons, the quasi-perceptual properties of the resulting images may be epiphenomenal with respect to the comparison process.

Similarly, Paivio's (1975) finding that size comparisons can be made more quickly when the items are presented as pictures rather than object names does not directly implicate imagery in the comparison process. It is only necessary to assume that pictures activate perceptual information in long-term memory more quickly than do words. It need not be the case that this perceptual information is either stored or processed in the form of images. 
This third hypothesis does not explain the similarity of the distance effect found in symbolic and perceptual comparisons. However, it suggests that the particular function found may be peculiar not to perceptual and quasi-perceptual comparisons of entire objects, but rather to analog comparisons in general. Accordingly, the explanation for the parallels between perception and memory should emerge with a general model of magnitude comparisons applicable to dimensions in either domain. A discussion of possible comparison models is provided by Holyoak and Walker (1976). It can be argued that the imagery hypothesis (hypothesis 1 above) is itself only a pseudo-explanation of the observed parallels. That hypothesis assumes that the effects obtained with perceptual comparisons should be obtained with imaginal comparisons as well, but does not directly explain why these effects should be obtained in either case.

\section{Design of the Experiments}

In the experiments to be reported, people were timed as they decided from memory which of two objects was the larger. If people comparc images in order to reach a decision, it should be possible to affect their decision latency by distorting the relative size of their images of the two objects (Experiment I) or by requiring them to maintain irrelevant images while making the size comparison (Experiment II). Both these two experiments involved two groups of subjects that differed in instructions; one group was told that they must use imagery to make the size comparisons, while the other group was free to make their decisions in any way possible. From what is known about the properties of images, it should be possible to predict how different experimental manipulations will affect decision time for subjects who are told to use imagery. If these variables then affect control subjects in the same way, it would suggest that they also use imagery to perform the task. But if the patterns of results differ between the two groups, the discrepancies may suggest a different account of how people make mental size comparisons when not directed to use imagery.

In addition, the objective difference between the sizes of the objects being compared was experimentally varied. If the role of imagery depends on how close in size the objects are, this distance factor should interact with variables that determine how easy it is to use imagery in the task.

\section{EXPERIMENT I}

On each trial in Experiment I, subjects were first told the name of an object and asked to image it either as large as possible, as small as possible, at its normal size, or not at all. They were then presented with the name of a second object and immediately had to determine which of the two objects was larger in the real world. If subjects make this decision by comparing images of two objects, then their initial image of the first object 
should affect their decision time. In order to make an accurate decision both objects must presumably be imaged at their canonical size. If the first object has been imaged larger or smaller than normal, the subject using imagery should need to change his initial image prior to the comparison. Similarly, if the subject initially did not form an explicit image of the first object, he should later have to construct a normal-sized image before he makes the size comparison. But if the subject initially formed a normalsized image, he should be ready to make the comparison without changing his internal representation of the first object. Accordingly, he should perform the task most quickly if he initially images the first object at its normal size.

In contrast, the advantage of a normal-sized image should be reduced or eliminated if the subject can make the size comparison on the basis of information more abstract than visual imagery. It is possible that forming an image of an object out of proportion to its true size will interfere with the subject's ability to retrieve the actual size, even if he is not using imagery to make the comparison. That is, thinking of a "medium-sized" object as very large or very small may simply be confusing. However, if the subject does not use imagery, then forming a normal-sized image should not lead to a faster decision than forming no image at all, which is also a semantically "neutral" condition.

Another possible effect of manipulating image size depends on the congruence of the size of the initial image with the correct decision. Paivio (1975) has shown that when objects are presented for a size comparison by means of pictures, RT is increased if the sizes of the pictured objects do not correspond to their relative size in the real world. For cxample, a person can decide that zebras are larger than lamps more quickly when the zebra rather than the lamp is pictured as the larger object. Similarly, in the present study a person might be able to make the correct decision more quickly if he initially imaged the zebra as large rather than small. This congruence effect depends on the relationship between competing sources of size information - in the present paradigm, image size and actual size. Thinking of an object as very large may interfere with deciding it is the smaller of two presented objects, while thinking of an object as very small may interfere with deciding it is the larger of the pair. This interference may well be semantic rather than strictly imaginal; i.e., imaging an object as large may be functionally equivalent to simply thinking of it as large. Accordingly, decision time should be faster if the size of the initial image of the first object matches rather than mismatches the correct response, regardless of whether the subject actually uses imagery to make the comparison.

The present study, unlike Paivio's picture experiment, allows us to distinguish between simple congruence of the initial image and the decision (which occurs when the first object is imaged at its normal size) and 
"supercongruence" (as occurs when the larger object is imaged as abnormally large, or the smaller object is imaged as abnormally small). If only the congruence effect ${ }^{1}$ operates, these latter conditions should produce even faster RT than the former. But if people actually are comparing canonical images to make a decision, normal-sized initial images should always facilitate the comparison process more than any nonnormal image.

To summarize these predictions: If subjects actually compare normalsized images of the two objects to evaluate relative size, they should respond most quickly if their initial image of the first object is normal. The no-image and matching size conditions (when size of image matches the correct response) should lead to slower decisions. The relative difficulty of the latter two conditions is not predicted. The mismatching size condition (when size of image does not match the correct response) should be slowest of all. In contrast, if subjects do not use imagery to make the comparison, then the only clear prediction is an increase in RT for the mismatching size condition. The normal-sized image condition should produce no greater advantage than the no-image condition.

\section{Method}

Sixty-four pairs of object names were selected from the norms provided by Paivio (1975). These norms provide the mean size rating of each item (on a 1-9 scale) based on responses from 49 subjects. Thirty-two of the pairs contained items as close as possible in normative size, ${ }^{2}$ subject to the constraint that three graduate students agreed on the relative size of each pair. The other thirty-two pairs contained items extremely different in size. Paivio (1975) has shown that the ratio of the size of the larger to the smaller object, derived from his normative size ratings, is a good predictor of comparison latency. The mean size ratio of the close pairs used in the present study was 1.06 (range, 1.01 to 1.17), while for the distant pairs the mean was 4.04 (range, 2.16 and 6.96).

Subjects were tested individually using a tachistoscope. All subjects received all four manipulations of the initial image of the first object. Before each trial, the experimenter said one of four words, "big," "normal," "small," or "first," to indicate the condition for the next trial. The subject then pressed a "start" button that initiated display of the first word at the top of the viewer. At the beginning of the experiment the subject was told that "big" meant he was to image the first object as large as he possibly could (illustrated by a line-drawn picture of a cat occupying virtually all of a $7 \times 10$-in. rectangle on a piece of paper); "small" meant he was to image the object as small as possible while still being able to see it (illustrated by a drawing of a cat occupying approximately $1 \times 0.75 \mathrm{in}$. in the center of the $7 \times 10$-in rectangle); "normal" meant he was to image the object at whatever size seemed most natural (illustrated by a cat occupying about $2 \times 3$ in. in the center of the rectangle); and "first" (standing for "first word") meant that he should read the first word but try not to form an explicit image of the object at any size. He was told

${ }^{1}$ The congruence effect discussed here is not to be confused with the kind of congruity effect that depends on the form of the comparative (larger vs smaller) used in the question (Jamieson \& Petrusic, 1975).

${ }^{2}$ Selecting very close items was recommended in a working paper by Kosslyn, Murphy, Bemesderfer, and Feinstein. 
that in this condition he should not worry if an image came "automatically," but to avoid making a deliberate effort to form an image.

On each trial the subject had $5 \mathrm{sec}$ to prepare appropriately with the first word. The first word then disappeared and the second word appeared in a position below that of the first word. The subject was then timed as he chose the larger of the two objects, pressing either an upper or lower response button depending on whether the top (first) or bottom (second) word named the larger object. The correct response (top or bottom button) always matched the position of the larger object. The subject was told that the correct response always depended only on the size of the objects in the real world, not in his image, and that he should consider only "normal or average-sized examples" of the two objects in making his decision. He was also told that some of the pairs were very close in size, so that his opinion of which object was normally larger might be different from that of the experimenter. He was assured that this was fine as long as he made the decision that he thought was correct.

While all subjects received the instructions described above, two descriptions of how to make the size comparisons were given to different groups of subjects. Subjects in the Control condition were told that it was essential that they be prepared with an image of the first object at the specified size (or no image) at the time the second word appeared, but that at this point they could do anything they like with the first image in order to make the comparison as rapidly as possible. These subjects were not told to use any particular technique in making the comparisons. Subjects in the Imagery condition were also told that it was critical to be prepared appropriately when the second word appeared, but also that they had to compare normal-sized images of the two objects on each trial before they responded. These subjects were told they could alter their image of the first object as soon as the second word appeared. In the "first" (no initial image) condition they were told to image both objects at their normal sizes as soon as the second word appeared. Imagery subjects were told that they should go through the process of normalizing and comparing images on each trial, even if they could have responded without doing so.

Major points of the instructions were repeated at least twice to all subjects. All were told that the experimenter was interested in "what effect, it any, the size of the first image or having an image has on how long it takes to make the size comparison" (adding, in the Imagery condition, "using the technique of normalizing and comparing images"), and that for this reason it was critical that the instructions were followed on each trial. Twenty practice trials illustrating all experimental conditions were then administered, after which the subject was questioned as to his compliance with instructions. The test items were then presented. Afterward, the subject was again questioned about what he thought he was doing. Subjects in the Imagery condition were asked to estimate the proportion of trials on which they actually compared normal-sized images of the two objects before responding. All subjects (with the exceptions noted below) appeared to understand the instructions readily, and each reported that he was virtually always ready with an image at the specified size when the second word appeared. Some subjects reported having difficulty in avoiding an image of the first object in the "first" (no-image) condition, but most reported that they at least did not form images as clear as those in the "normal" condition.

During the test trials, subjects were informed when they made a response indicating disagreement with the experimenter. When the subject reported that he actually disagreed, did not know the answer, or felt he had made an error, those facts were recorded.

Across subjects each word pair appeared equally often in each of the four initial-image conditions and in each of the two possible orders (so that the first item presented was equally often the larger and the smaller one). For any one subject, each pair occurred only once in a single condition, and the order of conditions in the test series was randomized.

Forty-eight Stanford undergraduates participated in the experiment either for pay or to satisfy a course requirement. Data from four other subjects tested in the Imagery condition were discarded. After the experiment, three gave indications they had not followed instruc- 
tions (two reported comparing images on less than $60 \%$ of the trials, and one reported not comparing images in the "first" condition). The fourth subject had a mean RT more than twice as long as the mean for other Imagery subjects.

\section{Results and Discussion}

Both subjects and word pairs were treated as random effects in all analyses of variance reported in the present paper. $F$ ratios for subjects and items were calculated separately and then combined to calculate minimum quasi- $F$ ratios, min $F^{\prime}$ (Clark, 1973). The assignment of items to treatments formed a latin-square design across subjects. To remove the effect of subject variability from the items $\times$ treatments interactions, the item analyses were done on difference scores between the mean RT for a particular item in a particular condition and the grand mean for all subjects in that latin-square group. All analyses were performed separately for Imagery and Control subjects.

Subjects did not produce the expected response on $0.7 \%$ of the distant pairs and $14.3 \%$ of the close pairs. They reported being mistaken on all the disputed trials for distant pairs and $4.4 \%$ of the close pairs. For $3.5 \%$ of the close pairs the subjects did not know the answer, and for $7.4 \%$ they actually disagreed with the experimenter. Including RTs for the latter type of response did not alter the pattern of results in any way. Accordingly, the analyses below include RTs for disagreements. Data from trials when the subject admitted an error or did not know the answer was dropped. The number of disputed trials was virtually the same for Imagery and Control subjects, although Imagery subjects tended to make fewer admitted errors ( 1.8 vs $3.3 \%)$.

The major RT results of the experiment are depicted in Fig. 1. Subjects in both instructional groups chose the larger object much more quickly for the pairs with large rather than small size differences, replicating the effect of symbolic distance obtained by Moyer (1973) and Paivio (1975). This RT difference was $493 \mathrm{msec}$ for the Imagery group, min $F^{\prime}(1,56)$ $=59.3, p<.001$, and $379 \mathrm{msec}$ for the Control group, $\min F^{\prime}(1,43)$ $=77.3, p<.001$. Overall, subjects in the Control group responded 638 msec more quickly than subjects in the Imagery group. The difference is not surprising, since instructions to the Imagery subjects stressed use of the imagery technique over speed. Imagery subjects not only had to use imagery to make their decisions, but also had to monitor their decision process to satisfy themselves that they were complying with instructions.

The results of major interest center on the manipulation of the initial image of the first object. As Fig. 1 indicates, this factor depended critically on the instructional condition. The results for the Imagery subjects are fully consistent with the view that they compared images of the two objects at their canonical sizes in order to reach a decision. The 


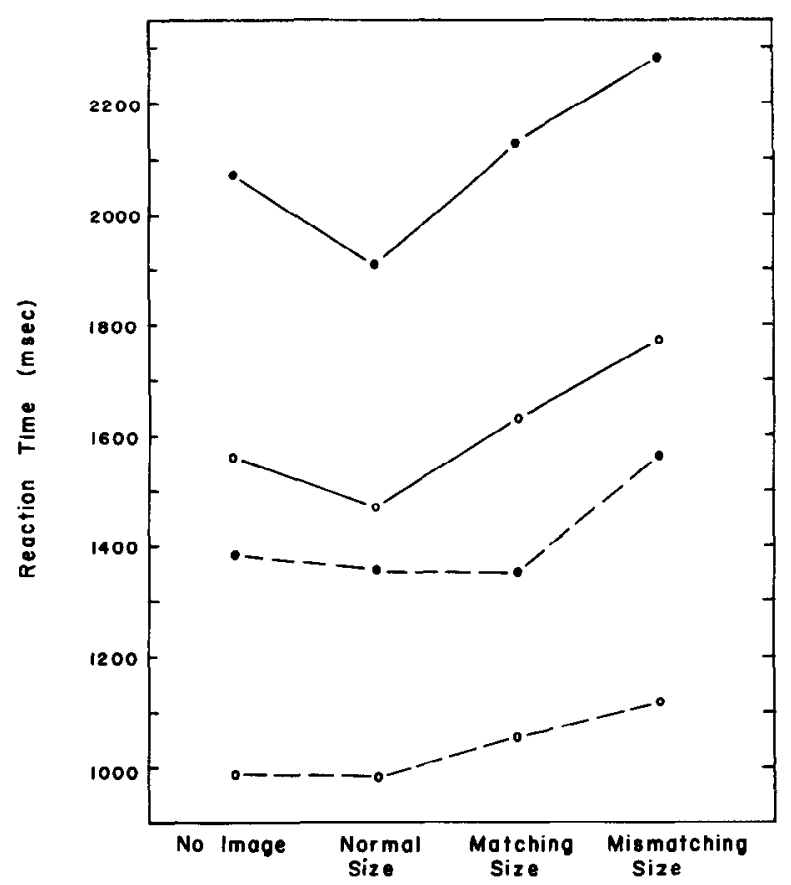

FIG. 1. Mean RT as a function of initial image of first object. Solid line, Imagery condition; broken line, Control condition; filled circle, small size difference; open circle, large difference.

overall differences among the four initial-image conditions were highly significant, $\min F^{\prime}(3,105)=10.6, p<.001$. Differences among the four conditions were tested using the Newman-Keuls method $(\alpha=.05)$. Imagery subjects responded significantly more quickly when prepared with a normal-sized image than in any other condition. The no-image condition was 123 msec slower than the normal condition, while the matching-size condition was $190 \mathrm{msec}$ slower. The difference between the latter two conditions was not significant. These results suggest that Imagery subjects actually performed mental operations to produce normal-sized images of the first object as soon as the second object was presented, changing the size of a nonnormal image or forming an image if they had not done so initially. Subjects in this group usually mentioned such processes when interviewed at the end of the experiment. Some subjects claimed to shrink or expand nonnormal images, while others claimed to simply replace them with a new image at normal size.

In addition, the Imagery subjects showed a strong congruence effect when the first object was imaged at a nonnormal size. The decision was $152 \mathrm{msec}$ faster when the size of the initial image matched the correct response (big when the first object was the larger, small when the first 
object was the smaller) than when the size mismatched the response. The latter condition was significantly slower than all of the other three treatments. The overall difference of $14 \mathrm{msec}$ between preparation with a big image vs small image was not significant. These results extend to internalized images the similar congruence effects that Paivio (1975) produced using pictures. But the congruence effect was clearly secondary for subjects in the Imagery condition, in that a normal-sized image of the first object produced faster mean RT than any nonnormal image size, even if the nonnormal size was congruent with the correct decision. ${ }^{3}$

The overall differences among the four initial-image conditions were also significant for the Control subjects, $\min F^{\prime}(3,126)=8.42, p<.001$. However, a Newman-Keuls test revealed a pattern very different from that obtained with Imagery instructions. A strong congruence effect was still evident: RT was $137 \mathrm{msec}$ faster when the size of the first image matched rather than mismatched the response. The latter condition was significantly slower than all three of the other conditions. However, no RT differences between the no-image, normal-sized, and matching-size conditions approached significance; the largest difference was a $38-\mathrm{msec}$ trend in favor of the normal over the matching size condition. It might be claimed that the no-image and normal conditions did not differ because subjects actually formed normal images in both conditions. However, the fact that an RT difference between these conditions was obtained with Imagery instructions argues against this interpretation. These results are consistent with the view that subjects normally do not compare images in order to decide which of two objects is larger, but rather compare abstract size parameters that are independent of the size of visual images constructed and maintained in active memory.

The present findings clearly do not support the hypothesis that imagery is always used in the size comparison process. Neither do they support the view that imagery is more likely to play an essential role in comparisons of objects very close in size. The effect of the various initial-image conditions did not differ significantly between the close and distant pairs, $\min F^{\prime}<1$ for the Imagery group, $\min F^{\prime}(3,131)=1.30, p>.20$, for the Control group. As Fig. 1 indicates, the trend in the Control group was actually opposite to the prediction of hypothesis 2: There was a greater RT difference between the normal and matching conditions for distant than for close pairs.

The only other RT difference that approached significance was an overall tendency for subjects to respond more quickly if the second item was the larger of the two. This difference was $82 \mathrm{msec}$ for the Imagery group, $\min F^{\prime}(1,67)=3.70, p<.10$, and $61 \mathrm{msec}$ for the Control group, $\min F^{\prime}(1,58)=3.29, p<.10$.

\footnotetext{
${ }^{3}$ These results for the normal size, matching size, and mismatching size conditions have been replicated with the item set used in Experiment II.
} 
We have assumed that the congruence effect-slower decision times when the initial size of the first object mismatches the correct decisionis to be interpreted as a type of associative interference. That is, thinking of an object as large conflicts with deciding it is the smaller, while thinking of an object as small conflicts with deciding it is the larger. Under this interpretation, the critical variable is the relation between the absolute size of the initial image and the relative size of the two objects. A possible alternative explanation based on imagery might claim that the critical variable is the relation between the absolute size of the initial image and the actual absolute size of the first object. For relatively large items (e.g., horse) the difference between a big image and normal will be less than the difference between a small image and normal, while for relatively small items (e.g., pencil), the difference between a small image and normal will be less than the difference between a large image and normal. If subjects shrink or expand nonnormal images to normal size before making size comparisons, then the closer the nonnormal image is to the normal size the faster the change may be effected. Accordingly, subjects should be relatively fast when their initial image is small if the first object is small in absolute terms and relatively fast when their initial image is big if the first object is large.

It turns out than on average, items that are the larger in a pair will be large in absolute terms, while items that are the smaller will be small in absolute terms. In the present study the mean rated size of the larger items was 5.18 , while the mean size of the smaller items was 2.87 . This raises the possibility that the congruence effect is actually due to the relative speed of transforming big vs small initial images to normal. To test this possibility the mean RT advantage of big over small initial images (which was negative when small was faster than big) was correlated with the rated size (from Paivio's, 1975, norms) of the imaged first object. This analysis was based on 128 cases ( 64 pairs in each of two orders). The imagery hypothesis just outlined predicts that this correlation will be significantly greater than zero. The obtained correlations were $r-.16$ for the Imagery group and $r=-.02$ for the Control group. Neither of these correlations was significantly different from zero. It therefore appears that for both Imagery and Control subjects the relatively slow RT for the mismatching size condition was due not to the time required to change a nonnormal image to normal size, but to associative interference between size of the initial image and the correct decision.

\section{EXPERIMENT ॥}

Whereas the previous experiment attempted to affect people's RT to make size comparisons by manipulating their image of one of the objects being compared, Experiment II introduced an irrelevant memory load to 
be maintained during the decision process. Prior to each trial, subjects were read three digits and were told either to rehearse the names of the digits covertly or to image the corresponding symbols. ${ }^{4}$ They then continued one of these two activities while comparing the size of two objects from memory. If a subject on a particular trial also uses imagery to make the size comparison, simultaneously imaging the digits should occupy processing capacity needed to reach a correct decision, and consequently should increase RT. Rehearsing the digits should presumably share less processing capacity with the imagery system, and hence produce less interference. On the other hand, if the subject performs the size comparison without making essential use of visual imagery, the effects of the two types of interference should be more equal. In particular, if the comparison is based on abstract analog size parameters, there is no reason to expect selective interference from either verbal or imaginal processing. The present design is thus similar to other selective interference paradigms used to investigate the role of imagery in other tasks (Brooks, 1968; Byrne, 1974; Elliott, 1973; Kosslyn, Holyoak, \& Huffman, 1976; Segal \& Fusella, 1970).

\section{Method}

Sixty pairs of names of common objects were selected for the experiment. Thirty of these are relatively close in objective size (e.g., pencil and cigarette). The other 30 pairs were created by replacing the terms in each of the close pairs by objects with a much greater size difference (e.g., typewriter and tack). Twenty Stanford students were asked to rate each pair on a 1-10 scale as to "how much difference in size you feel there is between the two objects," with a rating of 10 indicating maximal difference. For this task the pairs were listed in random order on a sheet of paper. The mean rating of the 30 close pairs was 3.64, and the mean rating of the 30 distant pairs was 7.92. The words in the two groups of pairs were balanced as closely as possible with respect to word length and frequency.

The same equipment was used as in Experiment $I$. The procedure was outlined to the subjects in detail at the beginning of the session. Before each trial, the experimenter read three randomly selected digits, and said either "Image" or "Rehearse." If he said "Image" the subject was to "picture the three digits in your mind as large and as clear as you can, in the order in which the experimenter says them." During the instructional phase the subject was shown a picture of three large digits side by side in a $7 \times 10$-in. rectangle to illustrate how the digits should be imaged. The instruction "Rehearse," on the other hand, was a cue for the subject "to repeat the names of the digits to yourself as rapidly as you can, over and over again." It was emphasized that both imaging and rehearsing were purely mental activities, e.g., the subject was not to repeat the digits aloud.

The subject was instructed to continue imaging or rehearsing for 3 or $4 \mathrm{sec}$ until the activity was clearly established, and then to press the "start" button to initiate the trial. After a 500-msec delay, the names of two objects appeared side by side in the viewer. The subject then had to press the left response button if the left object was the larger one, and the right button if the right object was larger. Each response was correct equally often across all test trials. The criteria for deciding which object was generally bigger were

${ }^{4}$ This selective interference paradigm has been used for a different purpose by Stephen Kosslyn (personal communication). 
TABLE 1

Mean RT to Decide Which Object Is Larger (EXPeriment II)

\begin{tabular}{lccccc}
\hline & \multicolumn{5}{c}{ Size difference } \\
\cline { 2 - 5 } & \multicolumn{2}{c}{ Small } & & Large \\
\cline { 2 - 5 } & $\begin{array}{c}\text { Image- } \\
\text { digits }\end{array}$ & $\begin{array}{c}\text { Rehearse- } \\
\text { digits }\end{array}$ & $\begin{array}{c}\text { Image- } \\
\text { digits }\end{array}$ & $\begin{array}{c}\text { Rehearse- } \\
\text { digits }\end{array}$ & $\bar{X}$ \\
\hline Imagery group & 2482 & 2248 & 2227 & 2048 & 2251 \\
Control group & 2108 & 2001 & 1806 & 1747 & 1916 \\
$\bar{X}$ & 2295 & 2125 & 2016 & 1898 & \\
\hline
\end{tabular}

outlined in the same way as in Experiment 1 . Subjects were told that it was critical that they try to continue imaging or rehearsing on each trial, even if it made their task more difficult.

All subjects rehearsed digits on half the trials and imaged digits on the other half. These trials were randomly interspersed. As in the previous experiment, half the subjects (the Imagery group) were instructed to compare images to evaluate the size of the pairs. They were told they could image the objects at the same time as the digits by imagining the objects superimposed on top of their images of the digits. The other subjects (the Control group) were not told any specific method of making their decisions.

Eighteen practice trials preceded the test trials, and as in Experiment I, subjects were questioned closely to ensure that they understood the instructions before the test trials began. At the end of the session, subjects were asked to estimate the proportion of trials on which they were able to continue rehearsing or imaging the digits until the final button press and also the proportion of trials on which they thought they used imagery to reach a decision. Any one subject saw each pair only once, in either the image-digit or the rehearse-digit condition, but across subjects all pairs appeared equally often in each interference condition.

Subjects were 36 Stanford undergraduates who participated either for pay or to satisfy a course requirement. Two other subjects tested under Imagery instructions reported being unable to image both digits and objects simultaneously, and their data were discarded.

\section{Results and Discussion}

The RT results of Experiment II are presented in Table 1. As in Experiment I, subject and item analyses were performed separately, with the latter being done on mean difference scores. Data from trials when the subject made errors $(3.8 \%)$ or disagreed with the designated correct response $(<1 \%)$ were dropped. The data for Imagery and Control subjects were combined into a single set of analyses.

As in the first experiment, subjects were able to decide which of two objects was the larger more quickly (by $253 \mathrm{msec}$ ) if the size difference was relatively large, $\min F^{\prime}(1,49)=35.6, p<.001$. In addition, subjects who were told to compare images in order to make the comparisons again responded more slowly (by $335 \mathrm{msec}$ ) than did Control subjects, min $F^{\prime}(1,33)=6.83, p<.025$. 
The main effect of interference type was also significant, $\min F^{\prime}(1,47)$ $=18.6, p<.001$. Imaging digits slowed the size comparison process more than did rehearsing digits. This overall difference in the difficulty of the two tasks is not surprising, since rehearsing digits is certainly a wellpracticed task, whereas imaging digits probably is not. In addition, the test pairs were presented visually, and imaging digits may have interfered to some extent with reading the words. As a result of this main effect, interactions involving type of interference are expressed as variations in how much extra interference was produced by imaging digits as opposed to rehearsing digits.

The only large variation of this sort involved the two instructional groups. Imaging digits slowed decision time $123 \mathrm{msec}$ more for the Imagery group than for the Control group. This interaction was reliable across the pairs used in the experiment, $F(1,29)=11.7, p<.001$, and also across subjects, $F(1,32)=4.23, p<.05$, although $\min F^{\prime}$ fell short of significance, $\min F^{\prime}(1,52)=3.10, .05<p<.10$. The effect of interference type was also tested separately for each instructional group. Imaging digits added $206 \mathrm{msec}$ to decision time in the Imagery group, a difference that was highly reliable, $\min F^{\prime}(1,50)=18.3, p<.001$, while this difference was reduced to a nonsignificant $83 \mathrm{msec}$ in the Control group, min $F^{\prime}(1,50)=2.96, .05<p<.10$. This result, like those of Experiment $I$, is consistent with the view that while people can compare images to evaluate relative size if instructed to do so, they otherwise make the decision using processes that are less dependent on visual imagery. This interpretation can only be made with caution, however, since it is possible that subjects in both groups were actually imaging, but Imagery subjects formed more detailed images and hence were more susceptible to imaginal interference. In addition, maximum interference was obtained when the more difficult secondary task (imaging digits) was combined with the more difficult primary task (making comparisons using imagery). It could be that the extra difficulty of these two tasks, rather than similarity of representations, was critical in producing selective inteference.

As in the previous experiment, there were no significant interactions between the degree of size difference and any other variable. The only trend of any note was a 54-msec tendency for imaging digits to produce more interference with the close than with the distant pairs. However, this effect fell far short of significance, $\min F^{\prime}(1,61)=1.59, p>.20$. Again, no strong evidence was found to support the hypothesis that imagery plays an especially important role in difficult size discrimination.

The only strong effect on errors was a higher error rate for the close pairs $(6.7 \%)$ than for the distant pairs $(1.1 \%)$. There were slightly more errors made by the Control than by the Imagery group (4.6 vs $3.0 \%$ ) and there were a few more errors made when rehearsing rather than imaging digits ( $4.3 \mathrm{vs} 3.4 \%$ ). Subjects in both instruction groups estimated that they 
were able to maintain the digits until the final button press on a somewhat greater percentage of the trials when the digits were rehearsed rather than imaged ( 85 vs $70 \%$ ). Subjects in the Imagery group estimated that they used imagery on $91 \%$ of the trials, while Control subjects gave a mean estimate of $59 \%$. These figures probably indicate little except that the Imagery subjects reported following instructions on most of the trials.

\section{GENERAL DISCUSSION}

\section{Summary and Implications}

The experiments reported here demonstrate that people, when instructed to do so, are able to compare images of objects in order to make size comparisons from memory. Subjects instructed to use imagery could perform the task more quickly if they prepared themselves with an image of one of the objects at its normal size, rather than with an image that was abnormally big or small, or no image at all. In addition, subjects using imagery to perform size comparisons were subject to substantial selective interference when asked to maintain irrelevant images of digits simultaneously. These effects are consistent with what is known about the structural properties of images, and therefore, argue that imageryinstructed subjects make essential use of imagery when performing mental size comparisons.

However, when people were not specifically instructed to use imagery to reach their decisions, the pattern of results changed radically. An initial image at normal size did not produce significantly faster decisions than no image, or a large or small image that was congruent with the correct decision. The only effect of image size shared by all subjects was the extra time required for decisions when the size of the initial image failed to match the correct decision. However, this congruence effect appeared to be due to associative interference rather than to imaginal processes. In addition, the sclective interference created by simultancously imaging irrelevant material was reduced for subjects not told to use imagery. Furthermore, the difficulty of the size discrimination did not interact with any other variable. The present study yields no support for the hypothesis that imagery is central to the size comparison process, even in decisions about objects very close in normative size.

While the present results suggest that size comparisons do not depend on visual imagery, they certainly do not decide the issue. As was pointed out earlier, it is possible that the difference between Imagery and Control subjects is essentially quantitative. Control subjects may use less detailed images, or rely on them less often, so that effects found with Imagery subjects are reduced in magnitude. The effects may become so small that the present designs were not sufficiently sensitive to detect them. Also, 
it is possible that people have multiple ways to perform size comparisons. The paradigms used here may have made it difficult for subjects to use imagery, causing the Control subjects to employ a mode of processing that is less susceptible to interference by the auxiliary task imposed upon them. People not given such auxiliary tasks may nevertheless prefer to use imagery. However, the results of Experiment I offer some difficulties for the latter argument. Preparing with a normal-sized image of the first object would seem unlikely to inhibit the use of imagery in the comparison process. Nevertheless, the normal-sized condition did not produce significantly faster decisions than the matching size or no-image conditions for Control subjects, even though the latter conditions produced more difficulty for Imagery subjects.

There are also limitations associated with the general method of comparing results obtained with subjects told to use a particular mental process to perform a task with the results for subjects not so instructed. First, it is necessary that subjects be able to introspect about the process in order to understand the instructions and then monitor their own compliance with them. Imaginal processes have seemed especially suited to the instructional method, since subjects generally act as if they know what it means to vary the size of images, to image different objects at once, or to image objects interacting in various ways (Kosslyn, 1975; Paivio, 1971). But one would hesitate to try and instruct subjects to "compare analog size parameters" or to "search your semantic network." Yet it may well be that the mental processes central to a cognitive task are simply not available to introspection.

Even if subjects are able to introspect about the mental processes they use to perform a task, it may be that the act of introspection itself alters task performance. If so, it is possible that instructed subjects will yicld a different pattern of results than control subjects even if all subjects use the same basic mental processes, simply because the instructed subjects are thinking about these processes as well as using them. Finally, if the instructed and control subjects are to produce the same pattern of results, the experimenter must accurately formulate the "natural" mental processes into the instructions he gives his subjects. But it is always possible that what appears to be a minor detail in the specified procedure is actually a critical determinant of whether the instructed subjects carry out the mental processes normally used in the task. For example, most subjects in the Imagery groups in the present experiments reported comparing the two objects by imaging them side by side. But it is possible that the "natural" comparison process involves superimposing the two images or imaging the objects sequentially. Had subjects been instructed to use some different variation of an imaginal comparison process, the results might have been altered. In general, if the instructed group does not perform in the same way as the control group, 
it is difficult to judge whether the differences are due to the radically different mental processes used by the two groups, or to minor variations in the same basic procedures.

\section{Further Issues}

While the present results are therefore only suggestive, the conclusion that finds most comfort is that visual imagery normally plays a less important role in the size comparison process than it does for imageryinstructed subjects. Yet all subjects, whether told to use imagery or not, were able to compare sizes much more quickly if the size difference between the objects was large. This argues that if people normally do not compare images to perform the task, they nonetheless do compare some more abstract representation of size that bears an analog relationship to the size of actual objects. The nature of this representation still largely remains an open question. But it now appears, to some extent contrary to initial expectations, that this problem is best approached by attempting to develop a general model of analog comparisons, rather than by asking detailed questions about the respresentation of magnitude for particular dimensions. The present negative conclusions about the role of imagery in size comparisons in fact support the possibility of a domain-independent model, since imagery seems an implausible vehicle for comparisons involving numerical magnitude (Moyer \& Landauer, 1967), or terms differing in degree along such dimensions as time and quality (Holyoak \& Walker, 1976).

The optimal formulation of such a general comparison model is open to question. However, available evidence suggests that it will include some form of a stochastic information-sampling procedure. The basic assumption is that a person can judge the relative magnitude of two concepts by comparing the absolute magnitude information stored with each. The comparison process will involve the continuous retrieval of magnitude information for each concept, so that the precision of available information will increase with time. Initial information will be sufficient to distinguish between concepts very different in magnitude (e.g., horse and cat), but more precise information (which takes more time to accumulate) will be needed to distinguish between concepts that are relatively similar in magnitude (e.g., horse and bear). The mechanism of such a comparison process might be formulated using various stochastic decision processes, such as a random walk (Buckley \& Gillman, 1974), accumulator (Pachella, 1974), or timing model (Luce \& Green, 1972). The central assumption of all such formulations, that information is aggregated over time, will yield a distance effect.

However, a number of important questions certainly remain in the domain of size comparisons. Is size represented as a single parameter, 
or is a size value for an object itself computed from more elementary dimensional values (i.e., for length, height, and width)? In the present experiments, as well as in previous studies of mental size comparisons, the objects to be compared usually have been chosen so as to be roughly comparable in shape and standard orientation. It is therefore possible that subjects in these experiments often judged relative size on the basis of a cornparison of magnitude along the most salient dimension (e.g., height). This suggests that additional computations may be required for size comparisons involving objects that are dissimilar in shape or orientation. People in fact report difficulty in comparing the size of objects such as a giraffe and an elephant, which differ in dominant axis. It is possible that visual imagery plays a much more active role in the size comparison process when it is necessary to form a global size estimate on the basis of the shape of the entire object.

Another question concerns the standards used to represent the size of objects in memory. If people directly or indirectly store information about absolute size, what are the units of measurement? Are objects measured relative to prototypical or salient exemplars of a superordinate (e.g., a mouse is a small animal), relative to some common standard used for all objects (e.g., the size of a person), or in multiple ways? Paivio's (1975) finding that comparisons between objects drawn from different semantic categories can be made just as quickly as comparisons between objects from the same category argues that at some level the size of all objects is represented on a common scale.

Finally, it is clear that people know something about the variability among category exemplars along different dimensions (Walker, 1975). Variability information might itself be stored as an analog value, or it might be computed on the basis of some process akin to the "availability" or "representativeness" heuristics (Kahneman \& Tversky, 1972; Tversky \& Kahneman, 1973). Issues such as these are likely to reward further exploration.

\section{REFERENCES}

Banks, W. P., Fujii, M., \& Kayra-Stuart, F. Semantic congruity effects in comparative judgments of magnitudes of digits. Journal of Experimental Psychology: Human Perception and Performance, 1976, 2, 435-447.

Brooks, L. R. Spatial and verbal components of the act of recall. Canadian Journal of Psychology, 1968, 22, 349-368.

Buckley, P. B., \& Gillman, C. B. Comparisons of digits and dot patterns. Journal of Experimental Psychology, 1974, 103, 1131-1136.

Byrne, B. Item concreteness vs spatial organization as predictors of visual imagery. Memory \& Cognition, 1974, 2, 53-59.

Clark, H. H. The language-as-fixed-effect fallacy: A critique of language statistics in psychological research. Journal of Verbal Learning and Verbal Behavior, 1973, 12, 335-359. 
Elliott, L. Imagery vs repetition encoding in short- and long-term memory. Journal of Experimental Psychology, 1973, 100, 270-276.

Holyoak, K. J., \& Walker, J. H. Subjective magnitude information in semantic orderings. Journal of Verbal Learning and Verbal Behavior, 1976, 15, 287-299.

Jamieson, D. G., \& Petrusic, W. M. Relational judgments with remembered stimuli. Perception \& Psychophysics, 1975, 18, 373-378.

Johnson, D. M. Confidence and speed in the two-category judgment. Archives of Psychology, 1939, No. 241, 1-52.

Kahneman, D., \& Tversky, A. Subjective probability: A judgment of representativeness. Cognitive Psychology, 1972, 3, 430-454.

Kosslyn, S. M. Information representation in visual images. Cognitive Psychology, 1975, 7, 341-370.

Kosslyn, S. M., Holyoak, K. J., and Huffman, C. S. A processing approach to the dualcoding hypothesis. Journal of Experimental Psychology: Human Learning and Memory, 1976, 2, 223-233.

Luce, R. D., \& Green, D. M. A neural timing theory for response times and the psychophysics of intensity. Psychological Review, 1972, 79, 14-57.

Moyer, R. S. Comparing objects in memory: Evidence suggesting an internal psychophysics. Perception \& Psychophysics, 1973, 13, 180-184.

Moyer, R. S., \& Bayer, R. H. Mental comparison and the symbolic distance effect. Cognitive Psychology, 1976, 8, 228-246.

Moyer, R. S., \& Landauer, T. K. Time required for judgments of numerical inequality. Nature (London) 1967, 215, 1519-1520.

Pachella, R. G. The interpretation of reaction time in information processing research. In B. H. Kantowitz (Ed.), Human information processing: Tutorials in performance and cognition. Hillsdale, NJ: Lawrence Erlbaum Associates, 1974. Pp. 41-82.

Paivio, A. Imagery and verbal processes. New York: Holt, Rinehart \& Winston, 1971.

Paivio, A. Perceptual comparisons through the mind's eye. Memory \& Cognition, 1975, 3, 635-647.

Palmer, S. E. Visual perception and world knowledge. In D. A. Norman, D. E. Rumelhart, \& the LNR Research Group (Eds.), Explorations in cognition. San Francisco, CA: W. H. Freeman, 1975.

Parkman, J. M. Temporal aspects of digit and letter inequality judgments. Journal of Experimental Psychology, 1971, 91, 191-205.

Potts, G. R. Information processing strategies used in the encoding of linear orderings. Journal of Verbal Learning and Verbal Behavior, 1972, 11, 727-740.

Potts, G. R. Storing and retrieving information about ordered relationships. Journal of Experimental Psychology, 1974, 103, 431-439.

Pylyshyn, Z. W. What the mind's eye tells the mind's brain: A critique of mental imagery. Psychological Bulletin, 1973, 80, 1-24.

Segal, S. J., \& Fusella, V. Influence of imaged pictures and sounds on detection of visual and auditory signals. Journal of Experimental Psychology, 1970, 83, 458-474.

Sekuler, R., Rubin, E., \& Armstrong, R. Processing numerical information: A choice time analysis. Journal of Experimental Psychology, 1971, 89, 75-80.

Tversky, A., \& Kahneman, D. Availability: A heuristic for judging frequency and probability. Cognitive Psychology, 1973, 5, 207-232.

Walker, J. H. Real-world variability, reasonableness judgments, and memory representations for concepts. Journal of Verbal Learning and Verbal Behavior, 1975, 14, 241-252. 